\title{
ANÁLISIS DE LA MAREA TERRESTRE GRAVIMÉTRICA EN LA ESTACIÓN SAN JUAN (ARGENTINA)
}

\author{
Analysis of the Gravimetric Earth Tide at the San Juan Station (Argentina)
}

\author{
Silvia Alicia Miranda ${ }^{1}$ \\ Cristian Ortiz ${ }^{1}$ \\ Alfredo Héctor Herrada ${ }^{2}$ \\ Maria Cristina Pacino ${ }^{3}$ \\ ${ }^{1}$ FCEFN, Universidad Nacional de San Juan. Meglioli 1160 Sur. J5400ARL Rivadavia, San Juan, \\ Argentina.Email: smiranda@unsj-cuim.edu.ar.Email:co.geof87@gmail.com \\ ${ }^{2}$ Facultad de Ingeniería, Universidad Nacional de San Juan. Av. Libertador Gral. San Martín 1109 Este, \\ J5400ARL San Juan, Argentina. Email: aherrada@uolsinectis.com.ar \\ ${ }^{3}$ CONICET- Universidad Nacional de Rosario. Av. Pellegrini 250, S2000BTP Rosario, Argentina. Email: \\ mpacino@fceia.unr.edu.ar
}

\begin{abstract}
Resumen:
Las mediciones de gravedad en la estación San Juan con un gravímetro automático Scintrex CG5 fueron analizadas con el propósito de obtener los parámetros de amplitud y diferencia de fase de mareas terrestres. Las observaciones abarcan un número total de 35 días. Para esta locación, este es el primer análisis de marea basado en mediciones de gravedad. El desarrollo de Tamura fue usado para el potencial de marea normal. Los parámetros de marea fueron estimados y analizados con el foco sobre las ondas de marea diurna y semidiurna. Se obtuvo un error cuadrático medio en la unidad de peso de $13,548 \mathrm{~nm} / \mathrm{s}^{-2}$ como resultado de un procedimiento de ajuste tomando en cuenta los cambios de presión barométrica. Los factores de amplitud computados para los principales constituyentes de onda fueron comparados con los parámetros correspondientes según la respuesta de marea sólida para un modelo de Tierra inelástica no hidrostática de Dehant, Defraigne y Wahr (DDW). Los factores gravimétricos locales calculados difieren hasta $\cong 2,7 \%$ del modelo DDW.
\end{abstract}

Palabras-clave: parámetros de marea-mediciones de gravedad

\begin{abstract}
:
Gravity measurements at the San Juan station with a Scintrex Automated CG-5 gravity meter were analyzed in order to obtain the Earth tidal parameters of amplitude and phase-difference. The observations cover a total number of 35 days. This is the first tidal analysis based on gravity measurements for this location. The Tamura tidal development was used as normal tidal potential. Tidal parameters were estimated and analyzed with the focus on diurnal and semidiurnal tidal waves. A root mean square error on the unit weight of $13.548 \mathrm{~nm} \mathrm{~s}^{-2}$ was obtained as a result of the adjustment procedure taking into account the barometric pressure changes. The
\end{abstract}


computed amplitude factors for the main wave constituents were compared with the corresponding parameters according to the response from the Dehant, Defraigne and Wahr (DDW) inelastic non hydrostatic Earth model. The computed local gravimetric factors differ by up to $\sim 2.7$ per cent from the DDW model.

Keywords: tidal parameters-gravity measurements

\section{Introducción}

La masa y los movimientos de la luna, el sol y en menor medida otros planetas inducen una variación del campo gravitatorio terrestre medido sobre la superficie. Además, debido a que la Tierra no es rígida se deforma de acuerdo a las propiedades elásticas locales, de modo que la distancia entre un punto en la superficie terrestre y el centro de la tierra es variable en el tiempo. Este es el efecto lunisolar que alcanza un máximo pico a pico de alrededor de $250 \mu \mathrm{Gal}$, y se caracteriza por ser periódico y en gran magnitud predecible. Las aproximaciones del potencial de marea pueden hacerse de una manera directa usando 1) las efemérides de la luna y el sol (y de otros planetas, si ellos son de interés), o 2) mediante una expansión en serie de armónicos esféricos. Ejemplo de 1) es el conocido algoritmo de Longman (1959), de gran difusión en exploración geofísica y cuyo código está incorporado en el microprocesador del gravímetro Scintrex CG-5. Para 2) se pueden mencionar los desarrollos de Cartwright-Tayler-Edden (1971; 1973) de 505 constituyentes y de Tamura (1987) de 1200 armónicos. A su vez, los parámetros de marea teóricos se obtienen calculando la respuesta a este potencial de marea de diferentes modelos de Tierra (sin océanos) (Dehant et al., 1999).

Superpuesto al denominado efecto directo descrito, se observa un efecto indirecto atribuido a la carga oceánica el cual representa en el caso de la aceleración de la gravedad un $10 \%$ del total de la marea observada (amplitud de hasta decenas de microgales). El efecto indirecto es complicado de predecir porque las mareas oceánicas son muy irregulares, y si bien tienen las mismas frecuencias, están desfasadas con respecto a las mareas terrestres.

Además de la mareas de la Tierra sólida y por la carga oceánica, las variaciones de la presión atmosférica ambiente también contribuyen a los cambios de gravedad en las frecuencias de marea solar y variaciones aleatorias (Spratt, 1982).

En la actualidad las mediciones relativas de gravedad con gravímetro Scintrex CG-5 pueden alcanzar precisiones de $3 \mu \mathrm{Gal}$ y exactitudes menores a $5 \mu \mathrm{Gal}$ (Christiansen et al., 2011), dependiendo en gran medida de la metodología de adquisición de los datos e incluyendo correcciones precisas. El efecto de marea lunisolar es la corrección más importante para estas lecturas de gravedad. En muchas aplicaciones la corrección lunisolar es aproximada considerando parámetros de marea constantes usando el algoritmo de Longman (1959). Por otro lado, en el caso de las redes gravimétricas con distintos fines, mediciones de gravedad absoluta y en microgravimetría aplicada a estudios de ingeniería, medio ambiente o geodinámica, donde se requiere de una mayor precisión, los cambios de la gravedad debido a las mareas terrestres necesitan ser eliminados con una precisión de 0,1 \% de su amplitud (Ducarme, 2009). De esta manera, la aproximación de Longman no es suficiente en estas aplicaciones (Bonvalot et al., 1998; Debeglia y Dupont, 2002). Por ejemplo, para una estación ubicada en San Juan, Miranda et al. (2013) encontraron diferencias de hasta $13 \mu \mathrm{Gal}$ en la marea predicha por modelos precisos (Tamura, 1987) y uno más simple como el de Longman. Debe tenerse en cuenta que la fórmula de Longman (1959) computa la atracción de marea gravitacional de la luna y el sol para un modelo de tierra rígida, y para tener en cuenta la elasticidad de la tierra sólida, los algoritmos 
suelen considerar un factor gravimétrico de amplificación de 1,16 (Scintrex Ltd., 2010), el cual es aplicado sin importar la latitud de la estación.

Además, en áreas cercanas a la costa el efecto de carga oceánica tiene que ser adecuadamente valorado para alcanzar precisiones del orden de algunos microgales (Debeglia y Dupont, 2002; Ducarme, 2009).

Los parámetros locales de marea pueden ser estimados a través de determinaciones continuas de gravedad. Es decir que, por medio del análisis de series temporales de gravedad es posible identificar distintas constituyentes de marea, con la ventaja de que los parámetros locales estimados con esta metodología ya integran la carga oceánica correspondiente. El instrumental más adecuado para estas mediciones son los gravímetros superconductores y los medidores de marea portátiles, tal como el gPhone (Micro-g LaCoste Inc.). Por otro lado, Ducarme y Somerhausen (1997) probaron que el gravímetro Scintrex es capaz de proveer parámetros de mareas confiables en las bandas diurna y semidiurna, Hasta el presente, en territorio argentino no se han realizado determinaciones sistemáticas de parámetros de marea locales de modo que, por ejemplo, en el procesamiento de la nueva red de gravedad de orden cero establecida recientemente (Lauría et al., 2014) se están usando parámetros de marea derivados de modelos teóricos. En este trabajo se presentan las estimacioes de los factores de amplitud y diferencia de fase de los principales constituyentes de marea diurna y semidiurna para la ciudad de San Juan. Para ello se usaron valores de gravedad correspondientes al registro continuo de 35 días de duración, medidos con un gravímetro relativo Scintrex CG-5. Los parámetros de marea se modelan mediante un ajuste mínimo cuadrático de los datos de aceleración de gravedad real y la presión atmosférica ambiente, de acuerdo al algoritmo ANALYZE (Wenzel, 1997). Los parámetros de marea modelados son consistentes con aquellos predichos por las aproximaciones teóricas.

\section{Adquisición de las series de tiempo de gravedad}

El gravímetro Scintrex CG-5 S/N 40484 fue puesto a registrar en modo continuo ("cycling mode") durante 35 días (14 de junio hasta 19 de julio de 2011) en la ciudad de San Juan, específicamente en dependencias de la Facultad de Ciencias Exactas, Físicas y Naturales de la

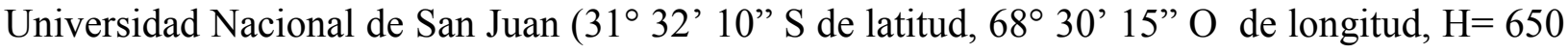
$\mathrm{m}$, Figura 1). A unos $10 \mathrm{~km}$ al oeste se ubica la estación de gravedad absoluta Zonda, cuyo pilar se encuentra en el interior del edificio del Instituto Geofísico Sismológico Volponi. La estación Zonda integra la red de gravedad de orden cero de Argentina remedida en 2014 (Lauría et al., 2014).

Previamente, el gravímetro fue calibrado midiendo sobre una línea de calibración en dirección norte-sur (extensión de $\sim 7^{\circ}$ de latitud), con un rango de $\sim 732 \mathrm{mGal}$, entre los puntos de gravedad absoluta Zonda (Provincia de San Juan) y San Lorenzo (Provincia de Salta). El factor de escala lineal del equipo resultó ser 0,999441 $\pm 0,000024$, es decir con error relativo 2 × $10^{-5}$.

Para la medición continua de gravedad las lecturas se programaron con una duración de 85 segundos, sin corrección automática por marea terrestre ni deriva instrumental estática (Figura 2 a). El gravímetro utilizado tiene una deriva estática estable de alrededor de 8-12 $\mu \mathrm{Gal} / \mathrm{hora}$, que puede ser modelada adecuadamente, para el período considerado de 35 días, mediante un ajuste mínimo cuadrático de un polinomio de segundo grado exhibiendo un coeficiente de correlación de 0,99956 y residuos menores a $\pm 15 \mu \mathrm{Gal}$ (Miranda et al., 2013). Este 
comportamiento de la deriva ha sido monitoreado a través de 6 años de uso. La serie fue remuestreada a intervalos de 1 hora para coincidir con los registros de presión atmosférica ambiente local disponibles (Figura 2 b), los cuales fueron cedidos por el Servicio Meteorológico Nacional de Argentina. La Figura 2 c muestra las series de tiempo de gravedad una vez eliminada la deriva instrumental estática. Estas series representan principalmente, de acuerdo a la exactitud nominal de este equipo $(\sim 5 \mu \mathrm{Gal})$, a los distintos constituyentes de marea cuyos parámetros locales dependen primordialmente de la latitud y de las propiedades físicas de la tierra en esa locación. El espectro de amplitudes de las series de gravedad, obtenido usando el programa TSoft versión 2.2.5 (Van Camp y Vauterin, 2014), se observa en la Figura 2 d, en la cual se han indicado las principales ondas diurnas y semidiurnas identificadas en el registro.

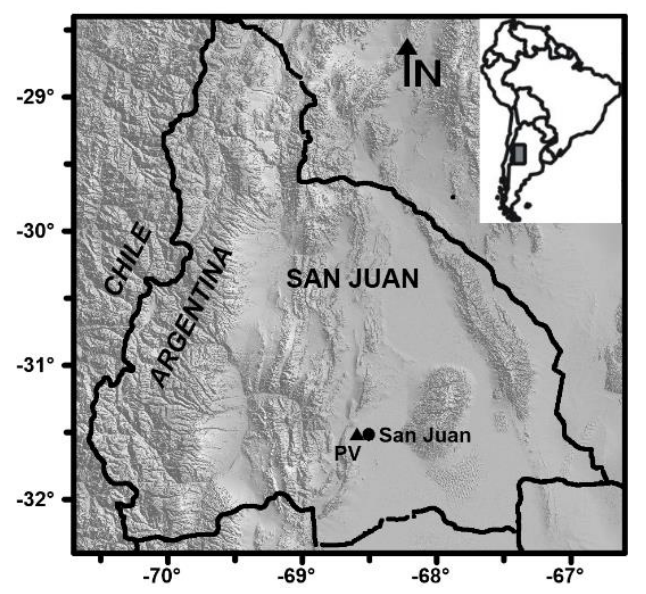

Figura 1: Mapa de ubicación de la estación San Juan (círculo sólido) en la cual se realizó la determinación de parámetros de marea. También se indica la localización de la estación de gravedad absoluta ubicada en el Instituto Geofísico Sismológico Volponi (triángulo sólido, PV).
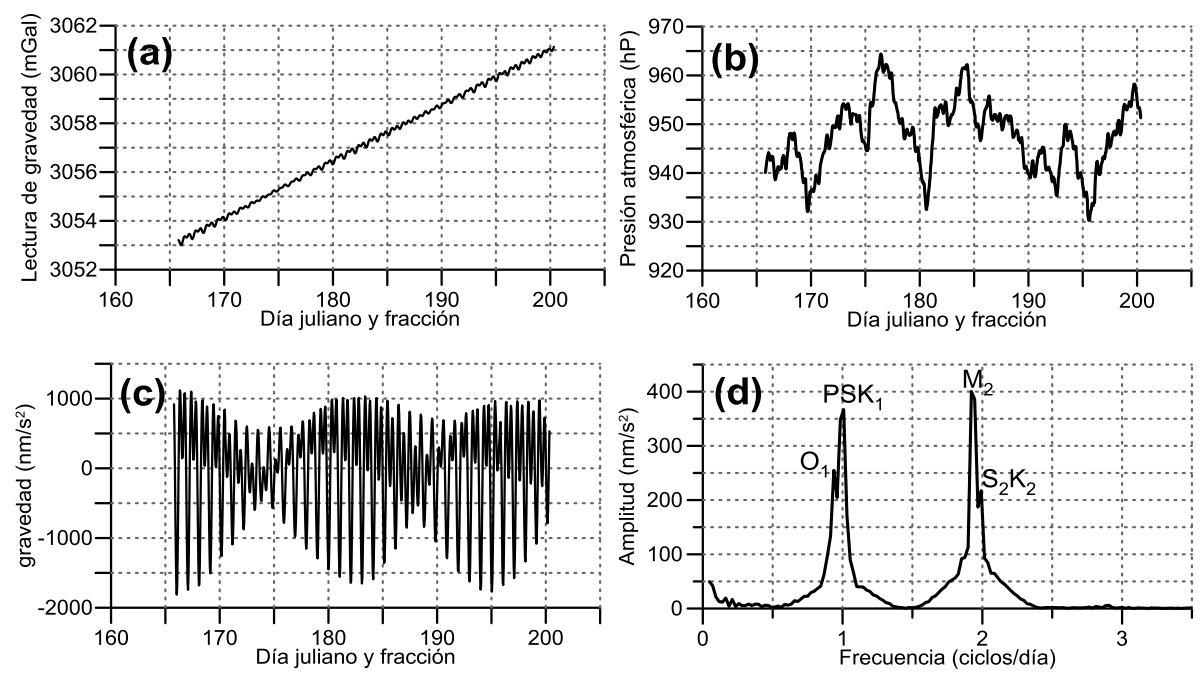

Figura 2: (a) Registro de lecturas de gravedad de 35 días de duración, adquiridas en la ciudad de San Juan. Nótese la tendencia positiva que representa la deriva estática del gravímetro

Scintrex CG-5 S/N 40484. (b) Presión atmosférica ambiente medida en San Juan entre el 14 de junio y el 19 de julio de 2011. (c) Marea terrestre observada. Se obtuvo eliminando de las lecturas medidas (Figura 2 a) un polinomio de segundo grado que sirvió para modelar la deriva estática $\Delta \mathrm{g}$ en función del tiempo $\mathrm{t}\left(\Delta \mathrm{g}=3007,8772+0,3080 \mathrm{t}-0,0002 \mathrm{t}^{2}\right)$. (d) Espectro de amplitudes de las series de gravedad en Figura 2c. Se indican las componentes diurnas $\left(\mathrm{O}_{1}\right.$,

$\left.\mathrm{PSK}_{1}\right)$ y semidiurnas $\left(\mathrm{M}_{2}, \mathrm{~S}_{2} \mathrm{~K}_{2}\right)$ identificadas. 


\section{Estimación de parámetros de marea}

El módulo ANALYZE del paquete de software ETERNA 3.4 (Wenzel, 1997) se usó para computar amplitudes, fases y factores de amplificación de los constituyentes de marea de gravedad. El análisis fue aplicado a las observaciones de gravedad y presión atmosférica, ambas muestreadas cada una hora (Figuras 2 c y 2 b, respectivamente). Los parámetros gravimétricos de marea, el factor de amplitud $\delta$ y la diferencia de fase $\alpha$, se estimaron en el rango de frecuencia de 0,50137 hasta 3,381378 cpd (ciclos por día).

En el análisis armónico se usó el potencial normal de Tamura (1987) con 1200 componentes, sin incluir ajustes por movimiento del polo y longitud del día (LOD), dado que estas correcciones se encuentran dentro del rango de ruido de la señal, propio del gravímetro utilizado. Se ensayaron varias soluciones, aplicando diferentes tipos de filtros que ANALYZE ofrece a los usuarios. En nuestro caso, la mejor solución se obtuvo sin necesidad de filtrado puesto que la deriva estática resultó apropiadamente modelada por el polinomio de segundo de grado.

Luego, ANALYZE determinó a través de un ajuste mínimo cuadrático los parámetros para los constituyentes de marea y un coeficiente de regresión lineal entre la presión atmosférica ambiente y la gravedad. Los resultados del análisis para 11 constituyentes de onda (6 diurnas, 4 semidiurnas, 1 tridiurna) se detallan en la Tabla 1. Esta solución presenta un error cuadrático medio en la unidad de peso de $13,548 \mathrm{nms}^{-2}$. El nivel de ruido medio calculado por ETERNA, obtenido a partir de los espectros de Fourier de los residuos de gravedad sin filtrar, es de 0,478 $\mathrm{nm} / \mathrm{s}^{2}$ y $1,315 \mathrm{~nm} / \mathrm{s}^{2}$ para las bandas diurna y semidiurna, respectivamente.

Tabla 1: Parámetros de marea y desviaciones estándar estimados a partir de la serie temporal de datos de gravedad para la estación San Juan. $\delta$ : factor de amplitud, $\alpha$ : diferencia de fase.

\begin{tabular}{c|c|c|c|c|c}
\hline \multicolumn{2}{c|}{ Onda } & \multicolumn{4}{c}{ Parámetros de marea } \\
\hline Nombre & $\begin{array}{c}\text { Amplitud } \\
\text { teórica }\left(\mathrm{nms}^{-2}\right)\end{array}$ & $\delta$ & $\begin{array}{c}\text { Desviación } \\
\text { estándar }\end{array}$ & $\begin{array}{c}\alpha \\
\left({ }^{\circ}\right)\end{array}$ & $\begin{array}{c}\text { Desviación } \\
\text { estándar }\end{array}$ \\
\hline Q1 & 52,9750 & 1,16176 & 0,02997 & $-0,6591$ & 0,8054 \\
\hline O1 & 276,6862 & 1,17149 & 0,02545 & 1,8019 & 0,1355 \\
\hline NO1 & 21,7604 & 1,17879 & 0,04144 & $-4,3598$ & 1,6016 \\
\hline PSK1 & 389,1311 & 1,16444 & 0,02533 & 1,1149 & 0,0775 \\
\hline J1 & 21,7598 & 1,13921 & 0,04760 & $-1,5665$ & 2,0189 \\
\hline OO1 & 11,9042 & 1,09234 & 0,07172 & 3,1969 & 3,4805 \\
\hline N2 & 104,4433 & 1,1584 & 0,02699 & $-0,0948$ & 0,3963 \\
\hline M2 & 545,5099 & 1,13239 & 0,02527 & 0,0129 & 0,0670 \\
\hline L2 & 15,4189 & 1,1756 & 0,09734 & $-7,2918$ & 4,6480 \\
\hline S2K2 & 253,7999 & 1,10179 & 0,02573 & $-0,4801$ & 0,2033 \\
\hline M3 & 9,1342 & 1,02429 & 0,07809 & $-3,1496$ & 4,1924 \\
\hline
\end{tabular}

El factor de admitancia de la presión atmosférica ambiente, estimado por ANALYZE junto con los parámetros de marea, es de $-4,226 \pm 0,162 \mathrm{nms}^{-2} / \mathrm{hPa}$, es decir que resulta dentro de los valores normales esperados $\left(-3 \ldots-5 \mathrm{nms}^{-2} / \mathrm{hPa}\right.$; Torge, 1989). 
Debemos hacer notar que seguramente de haber usado una serie temporal de mayor longitud hubiéramos conseguido disminuir los errores de la solución, identificar otros constituyentes y separar las ondas $\mathrm{S}_{2} \mathrm{y} \mathrm{k}_{2}$, por ejemplo.

\section{Comparación de los parámetros de marea estimados con parámetros sintéticos}

Los parámetros de marea determinados (Tabla 1) fueron comparados con los valores sintéticos predichos por el modelo inelástico no hidrostático de marea terrestre sólida WDD (Dehant et al., 1999). Para ello, los factores gravimétricos observados para las ondas principales (Tabla 1) fueron corregidos por efecto de carga oceánica. La expresión (1) (Ducarme, 2006) resume esta comparación, en la que cada término es formulado en coordenadas polares:

$$
\mathbf{A}_{\mathrm{c}}\left(\delta_{\mathrm{c}} \mathrm{A}, \alpha_{\mathrm{c}}\right)=\mathbf{A}\left(\delta \mathrm{A}_{\mathrm{t}}, \alpha\right)-\mathbf{L}(\mathrm{L}, \lambda) \approx \mathbf{R}\left(\delta_{\mathrm{t}} \mathrm{A}_{\mathrm{t}}, 0\right)
$$

Ac es la amplitud corregida, $\delta_{c}$ y $\alpha_{c}$ son el factor de amplitud y la diferencia de fase corregidos (Tabla 2). $\delta$ es el factor de amplitud (Tabla 1) que se define como el cociente entre la amplitud observada A y la amplitud teórica $A_{t}$ de la marea astronómica, $\left(\delta=\mathrm{A} / \mathrm{A}_{\mathrm{t}}\right)$. El vector observado de marea de gravedad $\mathbf{A}\left(\delta \mathrm{A}_{\mathrm{t}}, \alpha\right)$ es comparado con la respuesta teórica a la marea de un modelo de tierra inelástica no hidrostática, $\mathbf{R}\left(\delta_{t} A_{t}, 0\right)$. El vector $\mathbf{L}$ es la carga oceánica de amplitud $L$ y diferencia de fase $\lambda$.

Tabla 2: Se muestra la comparación, para los constituyentes principales de marea diurna y semidiurna, de los parámetros de marea del modelo inelástico no hidrostático de marea terrestre sólida, $\delta_{\mathrm{WDD} / \mathrm{NH}}($ Dehant et al., 1999) y los parámetros observados corregidos por la carga oceánica $\left(\delta_{\mathrm{c}}, \alpha_{\mathrm{c}}\right)$.

\begin{tabular}{c|c|c|c|c}
\hline Onda & $\delta_{\text {WDD } / \mathrm{NH}}$ & $\delta_{\mathrm{c.}}$ & $\begin{array}{c}\delta_{\mathrm{wDD}}-\delta_{\mathrm{c}} \\
\mathbf{( \% )}\end{array}$ & $\begin{array}{c}\boldsymbol{\alpha}_{\mathrm{c} .} \\
\left({ }^{\circ}\right)\end{array}$ \\
\hline $\mathrm{Q}_{1}$ & 1,15562 & 1,17489 & $-1,93$ & 1,8599 \\
\hline $\mathrm{O}_{1}$ & 1,15542 & 1,17503 & $-2,69$ & 2,4579 \\
\hline $\mathrm{N}_{2}$ & 1,15922 & 1,18233 & $-1,58$ & $-0,3711$ \\
\hline $\mathrm{M}_{2}$ & 1,15922 & 1,18442 & $-2,52$ & $-0,7558$ \\
\hline
\end{tabular}

Se usaron modelos de carga oceánica suministrados por el proveedor en línea del Observatorio Espacial Onsala de Suecia, administrado por M.S. Bos y H. G. Scherneck. Los modelos considerados fueron: DTU10 (Cheng y Andersen, 2010), EOT11a (Savcenko y Bosch, 2011), y HAMTIDE (Taguchi et al., 2014). La distancia de San Juan a la costa es superior a $500 \mathrm{~km}$ de modo que la carga oceánica es de reducida amplitud. Los distintos modelos predicen valores muy similares de carga oceánica (diferencias máximas de $0,02 \mathrm{~nm} / \mathrm{s}^{2}$ para las principales ondas), por lo cual se adoptó un valor promedio para cada uno de los constituyentes $\mathrm{M}_{2}, \mathrm{~N}_{2}, \mathrm{O}_{1}$ y $\mathrm{Q}_{1}$. La Tabla 2 muestra que los parámetros corregidos son consistentes con los valores teóricos respectivos (diferencia media: $2,2 \%$ ) para las ondas analizadas. 
Además, se calculó el vector de residuos, $\mathbf{X}$, el cual se define para un determinado armónico (e.g., $\mathbf{M}_{2}$ ) como la diferencia entre la amplitud observada $\left(\mathbf{A}_{\mathbf{c}}\right)$ y la estimada $(\mathbf{R})$ por un modelo teórico (e.g., DDW) a la que se ha descontado la atracción (L) calculada por un modelo de marea oceánica en particular, de acuerdo a la expresión (2) siguiente:

$$
\mathbf{X}(\mathrm{X}, \chi)=\mathbf{A}\left(\delta_{\mathrm{c}}, \alpha_{\mathrm{c}}\right)-\mathbf{R}(\mathrm{R}, 0)-\mathbf{L}(\mathrm{L}, \lambda)
$$

La componente del vector de residuos en fase con las ondas de marea de tierra sólida $(\mathrm{X} \cos \chi)$ sería sensible a heterogeneidades laterales y también es afectada por errores de calibración (Baker \& Boss, 2003). La componente en cuadratura (X sen $\chi$ ) refleja ruido instrumental y/o efectos no considerados en el modelo.

Los vectores residuos determinados para las ondas principales (Tabla 3), en especial para $\mathrm{M}_{2}$ y $\mathrm{O}_{1}$, indicarían tanto errores de calibración como ruido instrumental. Por otro lado, no se puede descartar alguna componente de carga oceánica no modelada. Cabe señalar que otra contribución a estos residuos podría provenir de heterogeneidades laterales en el manto superior debido al sistema de subducción subhorizontal entre la Placa de Nazca y la Placa Sudamericana, y a la topografía dinámica asociada con flujo en el manto (Métivier et al, 2008). Sin embargo, estas últimas producirían variaciones de gravedad de marea cuya magnitud es un orden de magnitud menor que la resolución del gravímetro Scintrex CG-5 aquí utilizado.

Tabla 3: Vectores residuos calculados para las principales constituyentes de ondas diurnas y semidiurnas.

\begin{tabular}{c|c|c|c|c}
\hline Onda & $\begin{array}{c}\mathbf{B} \\
\left(\mathbf{n m} / \mathbf{s}^{2}\right)\end{array}$ & $\begin{array}{c}\mathbf{X} \\
\left(\mathbf{n m} / \mathbf{s}^{2}\right)\end{array}$ & $\begin{array}{c}\mathbf{X} \cos \chi \\
\left(\mathbf{n m} / \mathbf{s}^{2}\right)\end{array}$ & $\begin{array}{c}\mathbf{X} \operatorname{sen} \chi \\
\left(\mathbf{n m} / \mathbf{s}^{2}\right)\end{array}$ \\
\hline $\mathrm{Q}_{1}$ & 0,67 & 2,25 & 0,99 & 2,02 \\
\hline $\mathrm{O}_{1}$ & 9,69 & 14,85 & 5,13 & 13,94 \\
\hline $\mathrm{N}_{2}$ & 0,19 & 2,54 & 2,41 & $-0,80$ \\
\hline $\mathrm{M}_{2}$ & 12,63 & 16,12 & 13,69 & $-8,52$ \\
\hline
\end{tabular}

\section{CONCLUSIONES}

La serie de tiempo de 35 días de las variaciones de gravedad obtenidas para San Juan con el gravímetro relativo Scintrex CG-5 S/N 40484 se usó para estimar parámetros de marea locales. Los factores de amplitud y diferencia de fase determinados para las componentes diurna y semidiurna son consistentes $(\sim 2,2 \%)$ con los parámetros teóricos para esta región. La precisión de estos parámetros seguramente será mejorada en determinaciones futuras usando una longitud de registro de al menos 6 meses, con el cual también podrán ser identificados otros constituyentes de onda. Para un futuro inmediato se está planificando a modo experimental la medición simultánea usando un gravímetro g-Phone (Universidad Nacional de San Pablo) y el Scintrex CG-5 de modo de obtener un mejor conocimiento del comportamiento de este último. También se planifica la determinación sistemática de parámetros de marea en distintas localidades de Argentina en las cuales se sitúan estaciones que forman parte de red de gravedad de orden cero. 


\section{REFERENCIAS BIBLIOGRÁFICAS}

Baker, T. F. and Bos M. S. "Validating Earth and ocean tide models using tidal gravity measurements". Geophys. Jour. Int. 152 (2003): 468-485.

Bonvalot, S., Diament, M. and Gabalda, G. "Continuous gravity recording with Scintrex CG3M meters: a promising tool for monitoring active zones". Geophys. Jour. Int., v. 135 (2) (1998): 470-494.

Cartwright, D. E. and Tayler, R. J. "New Computations of the Tide Generating Potential". Geophys. J. R. Astr. Soc. 23 (1971): 45-74.

Cartwright, D. E. and Edden A. C. "Corrected Table of Tidal Harmonics". Geophys. J. R. Astr. Soc. 33 (1973): 253-264.

Cheng, Y. and Andersen, O. B. "Improvement in global ocean tide model in shallow water regions". Paper presented at OSTST Meeting, Oct. 18-22, Lisbon, 2010.

Christiansen, L., Binning, P. J., Rosbjerg, D., Andersen, O. B. and Bauer-Gottwein, P. "Using time-lapse gravity for groundwater model calibration: An application to alluvial aquifer storage". Water Resour. Res. 47 (2011): 60-70.

Debeglia, N. and Dupont, F. "Some critical factors for engineering and environmental microgravity investigations". Jour. Appl. Geophys. 50 (2002): 435-454.

Dehant, V., Defraigne, P. and Wahr, J. "Tides for a convective Earth". Journal Geophys. Res. 104 B1 (1999): 1035-1058.

Ducarme, B. "Limitations of High Precision Tidal Prediction". Bull. Inf. Marées Terrestres 145 (2009): 11663-11677.

Ducarme, B. "Comparison of some tidal prediction programs and accuracy assessment of tidal gravity predictions”. Bull. Inf. Marées Terrestres 141 (2006): 11175-11184.

Ducarme, B. and Somerhausen, A. "Tidal gravity recording at Brussels with a Scintrex CG-3M gravity meter". Bull. Inform. Marees Terrestres 126 (1997): 9611-34.

Lauría, E., Cimbaro, S., Piñón, D., Miranda, S. A., Pacino, M. C. and Sanchez, R. E. "Towards a New Gravimetric network in Argentina". Paper presented at International Association of Geodesy Scientific Assembly, 150th Anniversary of the IAG, Sept. 1-6, 2013, Potsdam, Book of Abstracts (2013): 367.

Longman I. M. "Formulas for computing the tidal accelerations due to the Moon and Sun". Jour. Geophys. Res. 64 (12) (1959): 2351-2355.

Métivier, L. and Conrad, C. P. "Body tides of a convecting, laterally heterogeneous, and aspherical Earth”. J. Geophys. Res. 113 B11405 (2008), doi:10.1029/2007JB005448.

Miranda, S. A., Herrada, A. y Pacino, M. C. "Respuesta instrumental del gravímetro Scintrex CG-5 \#40484 en modos continuo y relevamiento". Revista Geoacta 38 (1) (2013): 1-14.

Savcenko, R. and Bosch, W. "EOT11a - a new tide model from Multi-Mission Altimetry". Presented at OSTST Meeting, Oct. 19-21, San Diego, 2011.

Scintrex Limited. CG5 Scintrex Autograv system Operation Manual. Scintrex Limited, Concord, Ont., Canada, 2010. 
Spratt R. S. "Modelling the effect of atmospheric pressure variations on gravity". Geophys. J. R. Astr. Soc. 71 (1982): 173-186.

Taguchi, E., Zahel, W. and Stammer, D. "Inferring deep ocean tidal energy dissipation from the global high-resolution data-assimilative HAMTIDE model". Jour. Geophys. Res. Oceans 119(7) (2014): 4573-4592.

Tamura, Y. "A harmonic development of the tide-generating potential". Bull. Inf; Marées Terrestres 99 (1987): 6813-6855.

Torge, W. Gravimetry. Walter de Gruyter Publishing Co., 1989.

Van Camp, M. and Vauterin, P. "Tsoft: graphical and interactive software for the analysis of time series and Earth tides". Computers \& Geosciences 31(5) (2005): 631-640.

Wenzel, H. G. Earth Tide Data Processing Package ETERNA 3.4. Manual ETERNA34.HLP, 1997.

Recebido em Abril de 2015.

Aceito em Julho de 2015. 